\title{
Energy and macronutrient intakes of Irish teenagers aged 13-17 years
}

\author{
J. Walton ${ }^{1}$, S. Doyle ${ }^{1}$, T. Joyce ${ }^{2}$, S. J. Burke ${ }^{2}$, E. M. Hannon ${ }^{1}$, M. J. Gibney ${ }^{2}$ and A. Flynn ${ }^{1}$ \\ ${ }^{1}$ Department of Food and Nutritional Science, University College Cork, Cork, Republic of Ireland and ${ }^{2}$ UCD Institute of \\ Food and Health, School of Agriculture, Food Science and Veterinary Medicine, University College Dublin, Belfield, \\ Dublin 4, Republic of Ireland
}

The objective of the present study was to estimate the energy and macronutrient intakes of Irish teenagers and to investigate their compliance with dietary recommendations. Analysis was based on The National Teens' Food Survey (NTFS), which was carried out between September 2005 and September 2006 to establish a database of habitual food and drink consumption in a representative sample of Irish teenagers aged 13-17 years. A 7 d semi-weighed-food record was used to collect food intake data from 441 teenagers (224 males, 217 females). Analysis of dietary intake data was carried out using WISPC (Tinuviel Software, Llanfechell, Anglesey, UK), which is based on McCance and Widdowson's The Composition of Foods, Sixth Edition ${ }^{(1)}$.

Mean daily energy intakes in males and females were 9.5 and 7.1 MJ respectively. Mean daily fat, saturated fat, carbohydrate and added sugar intakes (\% total energy; \%TE) were 35.6, 14.0, 49.0 and 12.4 respectively. Compliance with population goals ${ }^{(2)}$ was evaluated using the compliance method of Wearne \& Day ${ }^{(3)}$.

\begin{tabular}{|c|c|c|c|c|c|c|}
\hline & \multicolumn{6}{|c|}{ Compliers $(\%$ total $)$} \\
\hline & \multicolumn{3}{|c|}{ Males } & \multicolumn{3}{|c|}{ Females } \\
\hline & $\begin{array}{c}\text { All } \\
(n \text { 224) }\end{array}$ & $\begin{array}{c}\text { 13-14 years } \\
(n 95)\end{array}$ & $\begin{array}{c}\text { 15-17 years } \\
(n \text { 129) }\end{array}$ & $\begin{array}{c}\text { All } \\
(n \text { 217) }\end{array}$ & $\begin{array}{c}\text { 13-14 years } \\
(n 93)\end{array}$ & $\begin{array}{c}15-17 \text { years } \\
(n 124)\end{array}$ \\
\hline Fat $(\% \mathrm{TE} \leq 35)$ & 94 & 95 & 94 & 95 & 96 & 94 \\
\hline Saturated fat $(\% \mathrm{TE} \leq 11)$ & 30 & 25 & 33 & 33 & 26 & 38 \\
\hline Monounsaturated fat $(\% \mathrm{TE} \geq 13)$ & 58 & 59 & 57 & 58 & 59 & 57 \\
\hline Polyunsaturated fat (\%TE $\geq 6.5)$ & 56 & 58 & 54 & 65 & 58 & 71 \\
\hline Carbohydrate (\%TE $\geq 50)$ & 87 & 87 & 87 & 92 & 93 & 92 \\
\hline Added sugars $(\% \mathrm{TE} \leq 11)$ & 84 & 86 & 82 & 86 & 84 & 87 \\
\hline
\end{tabular}

The main food groups contributing to energy and macronutrient intakes are outlined below.

\begin{tabular}{ll}
\hline & \multicolumn{1}{c}{ Population $(n=441)$} \\
\hline Energy & Meat \& meat products $(16 \%)$, bread \& rolls $(12 \%)$, sugars, confectionery, preserves \& savouries $(12 \%)$ \\
Fat & Meat \& meat products $(22 \%)$, sugars, confectionery, preserves \& savouries $(13 \%)$, milk \& yoghurt $(12 \%)$ \\
Saturated fat & Milk \& yoghurt $(19 \%)$, meat \& meat products $(19 \%)$, sugars, confectionery, preserves \& savouries $(15 \%)$ \\
Monounsaturated fat & Meat \& meat products $(26 \%)$, sugars, confectionery, preserves \& savouries $(15 \%)$, potatoes \& potato products (11\%) \\
Polyunsaturated fat & Meat \& meat products $(19 \%)$, potatoes \& potato products (18\%), butter, spreading fats \& oils (13\%) \\
Carbohydrate & Bread \& rolls $(18 \%)$, sugars, confectionery, preserves \& savouries (13\%), potatoes \& potato products $(13 \%)$ \\
Added sugars & Sugars, confectionery, preserves \& savouries (32\%), beverages $(29 \%)$, breakfast cereals $(11 \%)$ \\
Protein & Meat \& meat products $(41 \%)$, milk \& yogurt $(13 \%)$, bread \& rolls $(11 \%)$ \\
\hline
\end{tabular}

At a population level, compliance with the UK goals for saturated fat, monounsaturated fat, polyunsaturated fat, carbohydrate and added sugars ranged from 30 to $93 \%$. Although compliance with the goal for total fat was $95 \%$ at a population level, $56 \%$ of Irish teenagers exceeded the US maximum recommended fat intake of $35 \% \mathrm{TE}^{(4)}$.

The project was funded by the Irish Government under the National Development Plan 2000-2006.

1. Food Standards Agency (2002) McCance \& Widdowson's The Composition of Foods, Sixth Edition. Cambridge: Royal Society of Chemistry.

2. Department of Health (1991) Dietary Reference Values for Food Energy and Nutrients for the United Kingdom. London: H. M. Stationery Office.

3. Wearne S \& Day M (1999) Br J Nutr 81, S119-S126.

4. Institute of Medicine, Food and Nutrition Board (2002) Dietary Reference Intakes for Energy, Carbohydrate, Fiber, Fat, Fatty Acids, Cholesterol, Protein and Amino Acids. Washington, DC: National Academy Press. 